\title{
Ultrasound biomicroscopy for the assessment of postoperative complications after congenital cataract surgery
}

\author{
Wensi Chen ${ }^{1 \#}$, Shiping $\mathrm{He}^{2 \#}$, Lanxiang $\mathrm{Hu}^{1}$, Jie Zhu ${ }^{1}$, Wanhua Xie ${ }^{1}$, Daoman Xiang ${ }^{1 \wedge}$ \\ ${ }^{1}$ Department of Pediatric Ophthalmology, Guangzhou Women and Children's Medical Center, Guangzhou Medical University, Guangzhou, China; \\ ${ }^{2}$ Department of Cataract, Aier Eye Hospital of Zhongshan Xiaolan, Zhongshan, China
}

\#These authors contributed equally to this work.

Correspondence to: Daoman Xiang. Department of Pediatric Ophthalmology, Guangzhou Women and Children's Medical Center, Guangzhou Medical University, Guangzhou 510230, China. Email: xiangdm35@126.com.

Background: The aim of the present study was to investigate the role of ultrasound biomicroscopy (UBM) in the evaluation of postoperative complications in children with congenital cataracts.

Methods: A retrospective study was conducted between September 2012 and December 2016 at Guangzhou Women and Children's Medical Center. Red reflex test and high-resolution bag/balloon UBM were performed to evaluate postoperative congenital cataracts. The red reflex test results were recorded, and UBM imaging results were recorded and analyzed. Different postoperative complications were classified based on the UBM imaging features, and a second procedure was performed accordingly. The UBM images were compared with the images captured from the intraoperative videos.

Results: In total, we looked at 120 eyes in 96 patients (65 males and 31 females) in the present study. The age of the cohort was 3-76 months. A total of 51 eyes with poor red reflex were included. There were complications in 46 eyes after congenital cataract surgery, as detected by UBM, including posterior capsular opacification ( $\mathrm{n}=29$ eyes), pupil block ( $\mathrm{n}=8$ eyes), synechia ( $\mathrm{n}=5$ eyes), hyphema ( $\mathrm{n}=1$ eye), and abnormal intraocular lens (IOL) placement ( $\mathrm{n}=3$ eyes). UBM images showed specific features of postoperative complications.

Conclusions: UBM is a valuable tool for the early evaluation of postoperative complications of congenital cataracts, especially for those with media opacities or when pupil dilation is not possible.

Keywords: Ultrasound biomicroscopy (UBM); congenital cataracts; postoperative complications; diagnosis

Submitted Aug 18, 2020. Accepted for publication Nov 16, 2020.

doi: $10.21037 /$ qims-20-185

View this article at: http://dx.doi.org/10.21037/qims-20-185

\section{Introduction}

Congenital cataracts are one of the most common treatable causes of childhood blindness. Approximately, 200,000 (10$30 \%)$ children worldwide are estimated to suffer cataractassociated blindness every year (1). The current standard of care for congenital cataracts involves surgical removal of the cataractous lens and implantation of an artificial intraocular lens (IOL), simultaneously or subsequently, to replace the missing refractive media. However, the postoperative complication rate of congenital cataract surgery is high, and even after successful congenital cataract surgery in children, various postoperative complications, such as pupil block, posterior cataract, synechia, abnormal IOL, are reported (2). The management of childhood cataracts is often difficult,

\footnotetext{
^ ORCID: 0000-0002-3474-8346.
} 
requiring frequent follow-up over several years.

The diagnosis and timely treatment of postoperative complications in patients with congenital cataracts constitute a vital part of management for preventing irreversible deprivation amblyopia (3). Slit-lamp examination and red reflex test were applied as the traditional and common methods for postoperative clinical evaluation. However, when corneal edema or pupils could not be dilated, it was difficult to evaluate the postoperative complications in infants for a comprehensive assessment of the anatomical change in their anterior segment.

Ultrasound biomicroscopy (UBM) is widely used to visualize the anterior portion of the globe at a microscopic resolution. Unlike the other optical methods, it is not limited by media opacity. In our previous study, preoperative UBM was performed to classify the anterior capsule and the cortex and posterior capsule of congenital cataracts (4). However, in the UBM examination, saline solution was filled in the eyecup, which might cause postoperative infection. To adapt UBM for early postoperative examination, a sterile water bag was used to replace the standard plastic shell, which would avoid any incision backflow caused by conjunctival sac fluid filling. Therefore, UBM could be valuable for the evaluation of postoperative anterior segment anatomy, especially posterior capsular opacification, synechia, pupil block, and abnormal IOL placement in children who undergo congenital cataract surgery.

\section{Methods}

\section{Patients and methods}

In the present retrospective study, data concerning the eyes of children with congenital cataracts and admitted to the Department of Ophthalmology, Guangzhou Women and Children's Medical Center, Guangzhou Medical University, from September 2012 to October 2016 were included for analysis.

Children diagnosed with congenital cataract by slitlamp microscopy, who were scheduled to undergo cataract surgery, were included in the study. Patients who had other previous ocular diseases or systemic diseases, did not have UBM, had a blurred UBM image, were not surgically confirmed cases, or who were lost to follow-up were excluded from the present study. A detailed history (including age and sex) was obtained from all patients, and a clinical ophthalmic examination before and after pupillary dilatation was conducted. The present study was approved by the Institutional Review Board/Ethics Committee of Guangzhou Women and Children's Medical Center (no. 40501), Guangzhou Medical University, and adhered to the principles of the Declaration of Helsinki. The present study was a retrospective trial; therefore, the requirement for informed consent was waived.

\section{Surgery and UBM examination}

All surgical procedures were performed by the same surgeon (DX), who has significant experience performing this procedure. The patients were placed under general anesthesia for extracapsular cataract extraction with anterior vitrectomy. Depending on age and preoperative assessment, some patients underwent IOL implantation (Aqua-SensePAL). The anterior segment was evaluated by UBM examination 2 weeks after the surgery, or if the red reflex test result was unclear.

For the UBM examination (Aviso S instrument; Quantel Medical, Cournon d'Auvergne, France), all patients were placed in the supine position under sedation with oral chloral hydrate and topical anesthesia with oxybuprocaine hydrochloride eye drops. The procedure was performed by an experienced technician (HU), who was positioned on the patient's right side. The patient's head was gently steadied by an assistant, and the eyes were studied by an ultrasonic probe equipped with a water bag, instead of the standard plastic shell (Quantel Medical, Cournon d'Auvergne, France). Any anatomical structural changes, such as iridocapsular synechiae, iridocorneal synechiae, iris defects, pupillary membranes, or lens remnants, were recorded. The UBM images were compared with those captured from intraoperative videos.

\section{Statistics}

All data were analyzed using SPSS version 23.0 (IBM, USA).

\section{Results}

In the present study, we looked at 153 eyes of 118 patients. A total of 33 eyes of 22 patients were excluded because of poor UBM scan quality and the inability to extract accurate data. Therefore, 120 eyes of 96 patients were analyzed. The age of the patients varied from 3 to 76 (median age: 15.5) months. Moreover, 24 patients were bilateral and 72 patients were unilateral. All patients underwent extracapsular cataract extraction with anterior vitrectomy; of these, 20 eyes (16.67\%) underwent primary 
Table 1 Baseline characteristics

\begin{tabular}{lc}
\hline Characteristics & Total $(\mathrm{n}=96)$ \\
\hline Females, $\mathrm{n}(\%)$ & $31(31.9)$ \\
Males, $\mathrm{n}(\%)$ & $65(68.1)$ \\
Age at diagnosis (months), median (minimum; & $15.5(3 ; 76)$ \\
maximum) & \\
Age at operation (months), median (minimum; & $7.3(2 ; 23)$ \\
maximum) & \\
Unilateral, $\mathrm{n}(\%)$ & $84(70.0)$ \\
$\begin{array}{l}\text { Bilateral, } \mathrm{n}(\%) \\
\text { Strabismus, } \mathrm{n}(\%)\end{array}$ & $18(30.0)$ \\
$\begin{array}{l}\text { Nystagmus, } \mathrm{n}(\%) \\
\text { Extracapsular cataract extraction with anterior }\end{array}$ & $0(0.0)$ \\
vitrectomy & $100 \%$ \\
IOL implantation & \\
$\quad$ The first time & \\
Secondary & \\
$\begin{array}{l}\text { Follow-up time from } 1 \text { to } 6 \text { months (months), } \\
\text { median (minimum; maximum) }\end{array}$ & $4.7(1 ; 6)$ \\
\hline
\end{tabular}

IOL, intraocular lens.

Table 2 Complications and prognoses

\begin{tabular}{lc}
\hline Complications & Total eyes $(\mathrm{n}=120), \mathrm{n}(\%)$ \\
\hline Total & $46(38.33)$ \\
Posterior capsular opacification & $29(24.17)$ \\
Hyphema & $1(0.83)$ \\
Synechia & $5(4.17)$ \\
Pupil block & $8(6.67)$ \\
Abnormal IOL placement & $3(2.50)$ \\
\hline
\end{tabular}

$\mathrm{IOL}$, intraocular lens.

IOL implantation in the capsule, and 79 eyes (65.83\%) underwent secondary IOL implantation in the ciliary sulcus or the capsule. Follow-up time from 1 to 6 months, median 4.7 (minimum 1, maximum 6) months (Table 1).

None of the patients developed infections after the UBM examination.

\section{UBM findings}

A total of 51 eyes with poor red reflex were tested with
UBM. Of these, 46 eyes (38.33\%) had complications, such as posterior capsular opacification $(\mathrm{n}=29$ eyes, $24.17 \%$ ), hyphema ( $\mathrm{n}=1$ eye, $0.83 \%)$, synechia ( $\mathrm{n}=5$ eyes, $4.17 \%$ ), pupil block ( $\mathrm{n}=8$ eyes, $6.67 \%$ ), and abnormal IOL placement ( $\mathrm{n}=3$ eyes, $2.5 \%$ ) (Table 2).

The typical UBM features of cases with or without postoperative complications in the present study are described.

\section{Cases without complications}

In total, 50 eyes (52.08\%) had no complications. Typical UBM imaging of the aphakic eye and IOL was different. The aphakic eye showed a clear cornea, normal anterior chamber depth, open anterior chamber angle, and normal visual axis area. The absence of the lens was seen as a hypoechoic zone (Figure 1A,B). The IOL also showed a clear cornea, normal anterior chamber depth, open anterior chamber angle, and normal visual axis area. The IOL was implanted in the ciliary sulcus or the capsule (Figure 1C,D).

\section{Pupil block}

In total, 8 eyes $(6.67 \%)$ had pupillary occlusion, causing secondary clinical glaucoma. UBM imaging showed iris distention and adherence to the cornea, whereas the anterior chamber disappeared (Figure $2 A$ ). The operating microscope revealed blurred cornea, and the anterior segment structure was ambiguous (Figure 2B). Cord sign hyperecho was detected in front of the IOL (Figure 2C). As shown in Figure 2D, another type of pupillary block was observed. Intraoperative images also showed cloudy cornea and blurred anterior segment structure (Figure 2D).

\section{Anterior synechia}

In the present study, the cornea of 5 eyes $(4.17 \%)$ appeared slightly blurred and edematous. In the UBM images, there was an uneven strong echo band in the anterior chamber in the iris image. Exudation could be seen on the iris surface. The iris echo belt was reflective and enhanced (Figure $2 A$ ).

\section{Anterior chamber bemorrhage}

Anterior chamber hemorrhage was observed in 1 eye $(0.83 \%)$. The UBM images of the same patient showed mild corneal edema. The anterior chamber was also filled with a consistent punctiform-like hyperecho (Figure $3 A$ ). It 

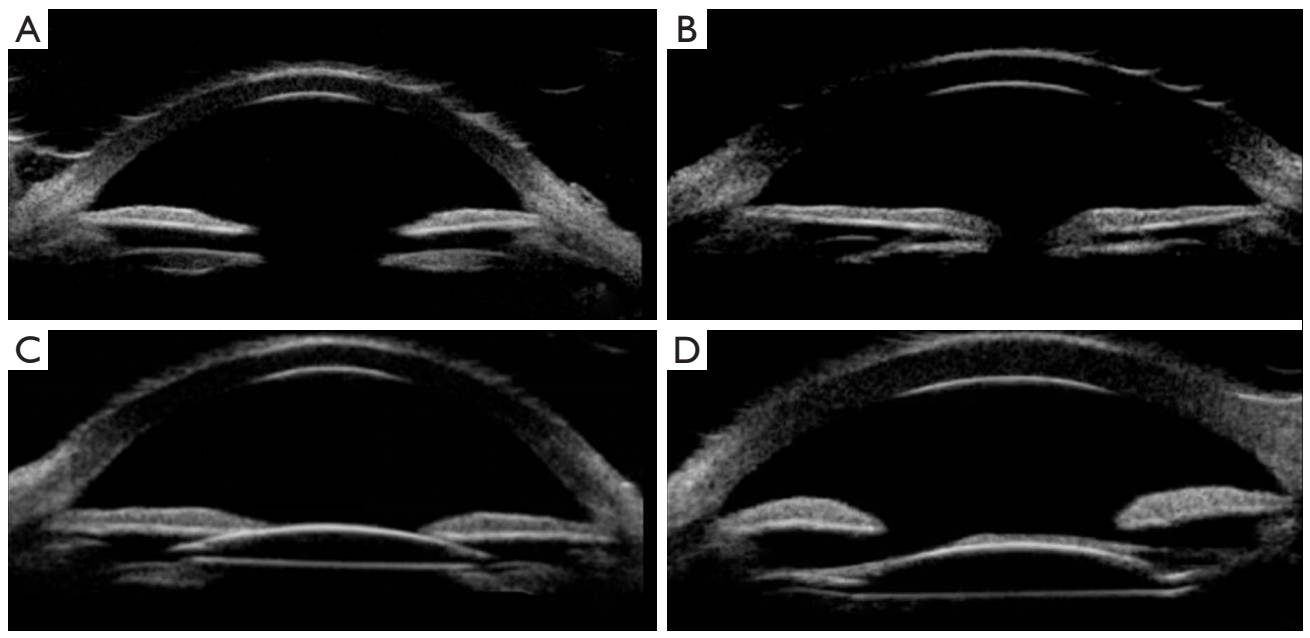

Figure 1 UBM imaging of cases with no complications. Both the aphakic eye and IOL had a clear cornea, normal anterior chamber depth, open anterior chamber angle, and normal visual axis area. Absence of the lens was seen as a hypoechoic zone (A,B). IOL was implanted in the ciliary sulcus (C) or the capsule (D). UBM, ultrasound biomicroscopy; IOL, intraocular lens.
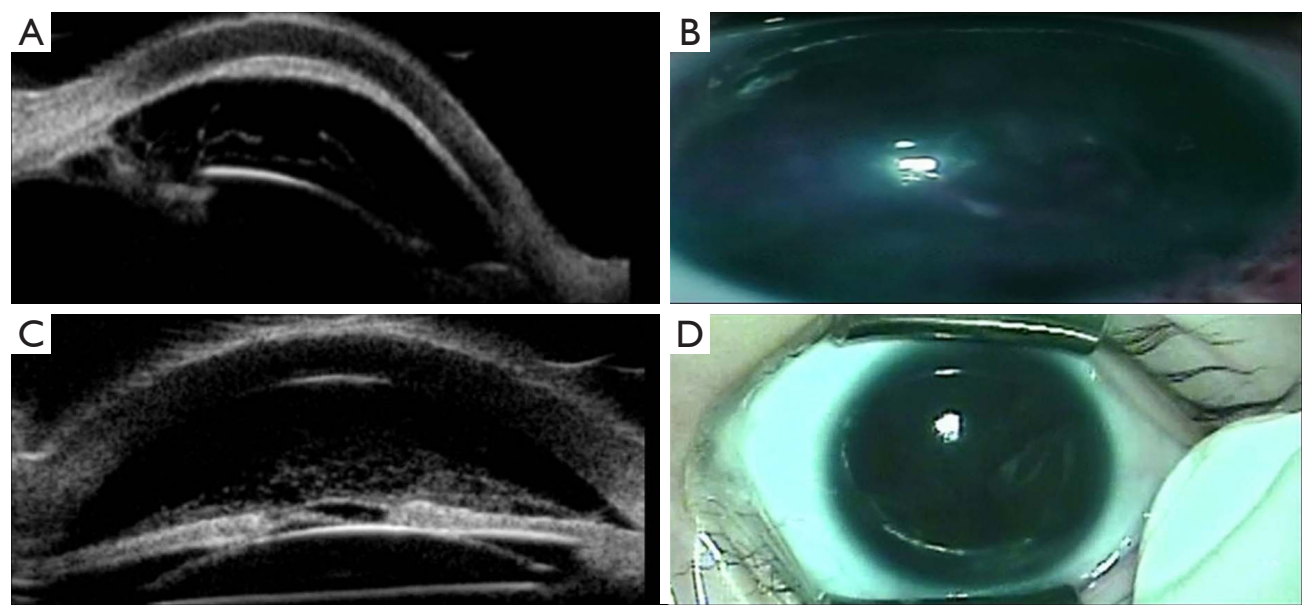

Figure 2 Comparison of UBM imaging with intraoperative videos of pupil block and anterior synechia. UBM imaging of pupil block showed iris distention and adherence to the cornea, and disappearance of the anterior chamber (A). Intraoperative videos of the pupil block showed that the cornea was blurred, and the anterior segment structure was ambiguous (B). Anterior synechia. UBM imaging showed that exudation could be seen on the iris surface. Iris echo belt was reflective and enhanced, and some cord sign hyperecho could be detected in the front of the IOL (C). Another type of pupillary block. Intraoperative images showed cloudy cornea and blurred anterior segment structure (D). UBM, ultrasound biomicroscopy; IOL, intraocular lens.

was difficult to observe the details of the anterior chamber by slit-lamp microscopy during bleeding (Figure 3B).

\section{Posterior capsular opacification}

Posterior capsular opacification was detected in 29 eyes (24.17\%). The UBM feature showed an intersection of the irregular patchy or cluster-like echoes and low echoes in the capsular bag. Continuous linear or spindle-shaped hyperechoic shadows were observed below the pupil area (Figure 3C). This phenomenon could not be checked by a slit-lamp microscope when the infants were awake; however, the white membrane of the pupil could be observed with surgical microscopy (Figure 3D). 

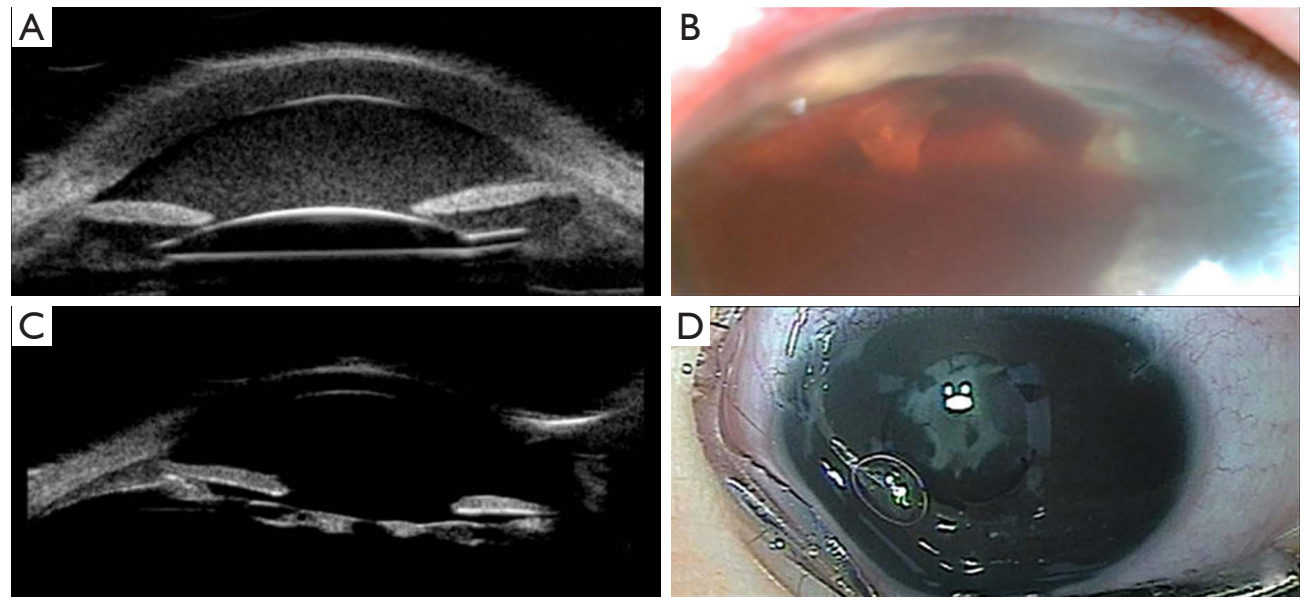

Figure 3 Comparison of UBM imaging with intraoperative videos of anterior chamber hemorrhage and posterior capsular opacification. Anterior chamber hemorrhage UBM image showed mild corneal edema and a consistent punctiform-like hyperecho (A). However, details could not be observed under a microscope (B). Posterior capsular opacification UBM image showed that, in the capsular bag, irregular patchy or cluster-like echoes and low echoes intersect with continuous linear or spindle-shaped hyperechoic shadows below the pupil area (C). White membrane of the pupil can be seen under a microscope (D). UBM, ultrasound biomicroscopy.
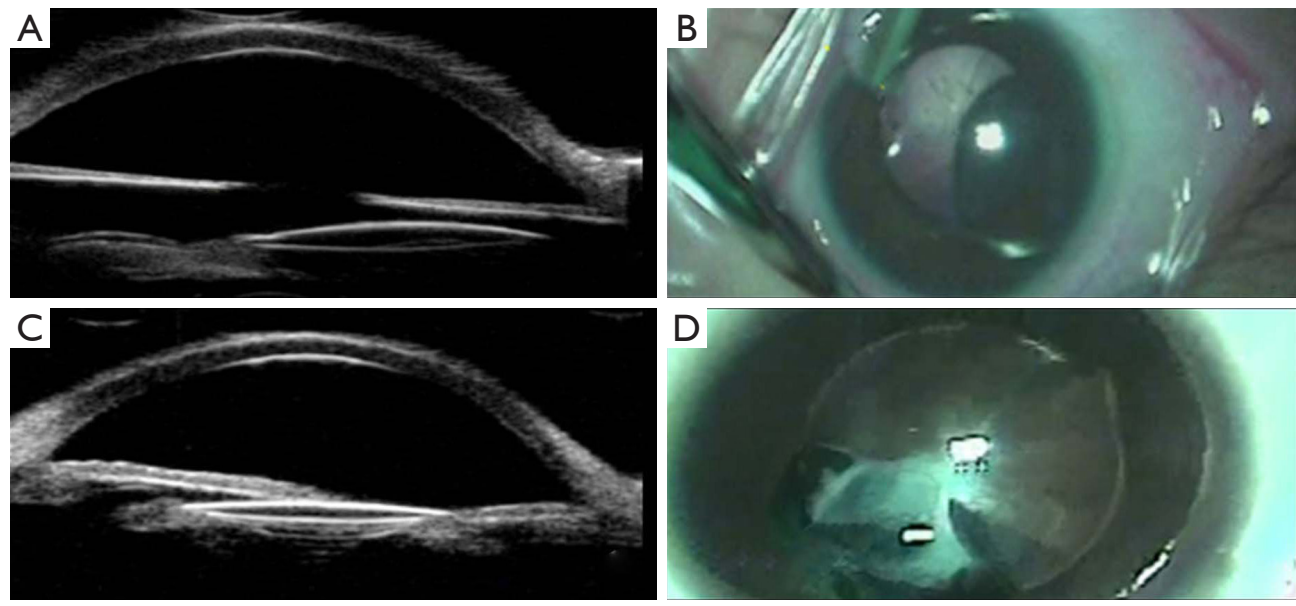

Figure 4 Comparison of UBM imaging with intraoperative videos of abnormal IOL placement. UBM showed that the IOL behind the iris shifted backward, and the nasal lateral and the temporal remained cortical behind; the IOL appeared to have mid-high echo (A). After the pupil was dilated, the shifted IOL could be seen under a surgical microscope (B). UBM image showed a high echo of the IOL, which was confined between the high iris echoes on both sides (C). IOL detail behind the iris could not be seen under a microscope (D). UBM, ultrasound biomicroscopy; IOL, intraocular lens.

\section{Abnormal IOL placement}

In the current study, 2 eyes (1.67\%) showed an IOL shift. UBM showed that the IOL shifted to the nasal side due to the proliferation of lens cortex, the IOL appeared as mid-high echo (Figure 4A). After the pupil was dilated, the shifted IOL could be seen under the surgical microscope
(Figure 4B). The UBM image showed high echo of the IOL, which was confined between the high iris echoes on both sides. The confined lens was the result of the compression of the regenerated cortex on both sides, seen as a high-echo area (Figure $4 C$ ). Iris-clip IOL was found in 1 eye $(0.83 \%)$. However, IOL detail behind the iris could not be detected easily by operation or a slit-lamp microscope (Figure 4D). 
Table 3 Results of red-light reflection were compared to the UBM images

\begin{tabular}{lcc}
\hline \multirow{2}{*}{ UBM } & \multicolumn{2}{c}{ Red reflex test } \\
\cline { 2 - 3 } & Normal & Abnormal \\
\hline Normal & 69 & 5 \\
Abnormal & 0 & 46 \\
\hline
\end{tabular}

UBM, ultrasound biomicroscopy.

\section{Red reflex test and UBM images}

A total of 69 eyes had clear red reflex, and 51 had poor red reflex. Complications after congenital cataract surgery were detected by UBM in 46 eyes (Table 3).

\section{Discussion}

Due to anatomical and physiological differences between the eyeballs of infants compared with adults, the preoperative evaluation, intraoperative operation, and postoperative management of infants with cataracts are more difficult to perform than those of adults $(5,6)$. Postoperative complications of congenital cataracts could also not be completely avoided (7).

Children who underwent surgery early in life had the highest number of outpatient visits. Frequent outpatient visits were required in the first year of life in one-fourth of the children who underwent bilateral surgery; in one-third of the children who underwent unilateral surgery, check-ups were required at least every 2 weeks (5).

Zhu et al. reported a case of UBM for secondary surgery of congenital cataract, following an intraoperative diagnosis of pupillary block-type secondary glaucoma (8). They found that UBM is an effective tool for the assessment of congenital cataract surgery; however, only 1 case of congenital cataract surgery of pupillary block-type secondary glaucoma was reported in their study. In our study, we found that the UBM can clarify the condition of the anterior chamber and find iris adhesion and the closed corner, under the condition of corneal opacity edema, so as to find the cause of secondary glaucoma after cataract surgery.

There are limited studies on the application of UBM in postoperative complications in patients with cataracts. In adult cataract patients, these can be checked easily using a slit lamp microscope. In cataract patients with severe postoperative complications, traditional UBM is not suitable because the eye cannot be soaked in water. The findings of the current study indicate that UBM has a high diagnostic value for pupil block, iris adhesion, and posterior capsular opacification in patients with complications due to cataract surgery.

The UBM imaging features of postoperative complications after congenital cataract surgery were as follows: a strong echo light belt with an uneven and coarse arc could be seen in the anterior chamber, seen as a medium-echo iris continuous image. The iris edge was not smooth, and the iris echo belt was reflective and enhanced. The posterior retinal cataract appeared as a hyperechoic shadow in the bilateral iris; a thin, linear, inhomogeneous medium hyperechoic shadow in the lens capsule; an irregular, lumpy, convex, medium hyperechoic shadow in the pupil region; or a normal, interrupted hypoechoic image in the pupil region.

In their study, Alniemi et al. used UBM following congenital cataract surgery to evaluate aphakia eyesight section shape and observe the lens capsule membrane remnants of the scope, situation of the lens capsule membrane and iris adhesion, guidance of the second phase of the surgical implant IOL, and posterior capsular residues, such as the phase II-implanted artificial crystal pendant (9). The authors postulated that UBM could effectively evaluate the absence of the lens after congenital defect cataract surgery and the cortical residue of the lens body in cases where posterior iris adhesion made it difficult to dilate the pupil, and observe the capsular membrane at the periphery of the lens. Previously published studies have reported that the UBM is used to describe the postoperative corner structure, measuring IOL implantation after anterior chamber depth (10). The IOL is highly reflective and can display the anterior and posterior interface of the IOL, the position of the IOL and its relationship with the surrounding tissues. Therefore, by using UBM to check whether the IOL is eccentric, tilted or dislocated, we can analyze the causes of postoperative complications of cataract surgery, provide reference for clinical data and guide the operation method.

Based on UBM imaging, postoperative complications related to congenital cataract surgery of infants could be observed, including cataract formation, the shape and thickness of the crystal residues in the pupil, the position correlation between the crystal residues and artificial crystal, classification of the crystal residue, and the correlation between the location of the iris. Novel surgical treatment modalities will be developed based on these findings. 
UBM is a valuable tool for the early evaluation of the postoperative complications of congenital cataracts. Using a UBM probe with a water bag, anterior segment structural changes could be assessed early. This will help with the diagnosis and treatment of postoperative complications, especially for those with media opacities or when pupil dilation is not possible.

\section{Acknowledgments}

Funding: The present study was supported by the Guangzhou Featured Project (no. 2019TS54).

\section{Footnote}

Conflicts of Interest: All authors have completed the ICMJE uniform disclosure form (available at http://dx.doi. org/10.21037/qims-20-185). The authors have no conflicts of interest to declare.

Ethical Statement: The present study was approved by the Institutional Review Board/Ethics Committee of Guangzhou Women and Children's Medical Center (no. 40501), Guangzhou Medical University, and adhered to the principles of the Declaration of Helsinki. This was a retrospective trial; therefore, the requirement for informed consent was waived.

Open Access Statement: This is an Open Access article distributed in accordance with the Creative Commons Attribution-NonCommercial-NoDerivs 4.0 International License (CC BY-NC-ND 4.0), which permits the noncommercial replication and distribution of the article with the strict proviso that no changes or edits are made and the original work is properly cited (including links to both the formal publication through the relevant DOI and the license). See: https://creativecommons.org/licenses/by-nc-nd/4.0/.

Cite this article as: Chen W, He S, Hu L, Zhu J, Xie W, Xiang D. Ultrasound biomicroscopy for the assessment of postoperative complications after congenital cataract surgery. Quant Imaging Med Surg 2021;11(4):1483-1489. doi: 10.21037/ qims-20-185

\section{References}

1. Muhit MA. Childhood cataract: home to hospital. Community Eye Health 2004;17:19-22.

2. Praveen MR, Vasavada AR, Shah SK, Khamar MB, Trivedi RH. Long-term postoperative outcomes after bilateral congenital cataract surgery in eyes with microphthalmos. J Cataract Refract Surg 2015;41:1910-8.

3. Lagrèze WA. The management of cataract in childhood. Klin Monbl Augenheilkd 2009;226:15-21.

4. Xiang D, Chen L, Hu L, Song S, Xie W, Long $\mathrm{J}$. Image features of lens opacity in pediatric cataracts using ultrasound biomicroscopy. J AAPOS 2016;20:519-22.e4.

5. Al-Bakri M, Sander B, Bach-Holm D, Larsen DA, Jensen H, Kessel L. Children with congenital and childhood cataract require frequent follow-up visits and examinations in general anaesthesia: considerations for the strain on families. Acta Ophthalmol 2019;97:778-83.

6. Medsinge A, Nischal KK. Pediatric cataract: challenges and future directions. Clin Ophthalmol 2015;9:77-90.

7. Whitman MC, Vanderveen DK. Complications of pediatric cataract surgery. Semin Ophthalmol 2014;29:414-20.

8. Zhu XJ, Zhang KK, He WW, Sun XH, Meng FR, Lu Y. Diagnosis of pupillary block glaucoma after removal of congenital cataracts with intraoperative ultrasound biomicroscopy: a case report. BMC Ophthalmol 2016;16:58.

9. Alniemi ST, Amin SR, Sculley L, Bakri SJ. Ultrasound biomicroscopy in pseudophakic patients with unexplained recurrent hyphema or vitreous hemorrhage. Semin Ophthalmol 2018;33:260-4.

10. Ang GS, Duncan L, Atta HR. Ultrasound biomicroscopic study of the stability of intraocular lens implants after phacoemulsification cataract surgery. Acta Ophthalmol 2012;90:168-72. 